\title{
Endoscopic Surveillance in Inflammatory Bowel Disease
}

\author{
Martin Goetz \\ Department of Internal Medicine I, University Hospital Tübingen, Tübingen, Germany
}

\begin{abstract}
Keywords
Inflammatory bowel disease - Crohn's disease .

Ulcerative colitis - Mucosal healing · Rutgeerts score ·

Dysplasia
\end{abstract}

\section{Summary}

Introduction: Endoscopy is a key technique for the surveillance of patients with inflammatory bowel diseases (Crohn's disease (CD) and ulcerative colitis). Indication to perform endoscopy for surveillance includes assessment of response to therapy, postoperative surveillance, and surveillance for intraepithelial neoplasia. Methods: In addition to personal experience, a literature search was performed and the guidelines were consulted. Results and Conclusion: Endoscopy is the gold standard in the long-term assessment of mucosal healing and in the short-term control of response to intensified therapy. For postoperative surveillance in $C D$, mucosal changes (as graded by the Rutgeerts score) are predictive of clinical recurrence. For neoplasia surveillance, virtual chromoendoscopy has not (yet) replaced conventional chromoendoscopy. Surveillance can be optimized by the use of high-definition endoscopes as well as (pan-)chromoendoscopy and requires time to scrutinize the mucosa. This approach seems to be superior to random biopsies.

(C) 2018 S. Karger GmbH, Freiburg

\section{Introduction}

When should patients with inflammatory bowel diseases (IBD) (Crohn's disease (CD) and ulcerative colitis (UC)) undergo endoscopic surveillance (table 1), i.e. repeat endoscopies for assessment of intraluminal pathologies? This review addresses the role of endoscopic surveillance in the following four clinical settings: surveillance for short-term control of response to intensified induction therapy; surveillance for mucosal healing as a sign of mid- to longterm therapeutic efficacy; surveillance for postoperative recurrence with the intent of stratification towards early versus late (or no) reintroduction of medical therapy; and surveillance for dysplasia in long-standing UC or Crohn's colitis. In these settings, endoscopy is the gold standard for mucosal assessment, complementing (but not replacing) clinical and laboratory data (including calprotectin) and cross-sectional imaging methods such as transabdominal ultrasonography or magnetic resonance imaging.

Initial diagnosis of the disease and its extent and behaviour as well as repeat endoscopies in patients with undefined enterocolitis are not within the focus of this review. Routine endoscopic reassessment is unnecessary for patients in clinical remission prior to inclusion in a dysplasia surveillance program, unless it is likely to change management. Such clinical situations may include refractory or relapsing disease, atypical symptoms, recurrent bleeding, and suspected infectious complications, also prior to surgery.

\section{Assessment of Mucosal Healing}

Mucosal healing is associated with a lower risk of clinical relapse, fewer hospitalisations, less need for proctocolectomy in UC, and lower risk of colitis-associated neoplasia, and may change the natural course of IBD. In most of the earlier studies on UC, mucosal healing has been defined as a Mayo endoscopic subscore of $0-1$. This score was developed by Schroeder et al. [1] in 1987, i.e., before high-definition video endoscopes became available for routine clinical practice. It is important to know that a subscore of 1 is defined as mild disease with erythema, decreased vascular pattern, and mild friability, and as such indicates ongoing low-intensity inflammation. A more rigid definition requires an endoscopic subscore of 0 . Newer scores such as the Ulcerative Colitis Endoscopic Index of Severity (UCEIS) evaluate the mucosal vascular pattern (normal, patchy, complete obliteration), bleeding (none, mucosal, luminal mild, luminal moderate or severe), and the presence of erosions and ulcers (none, erosions, superficial, deep) [2]. This score has been found useful to predict the clinical recurrence rate

\section{KARGER}

() 2018 S. Karger GmbH, Freiburg

Fax +497614520714 
Table 1. Indication for surveillance colonoscopy in inflammatory bowel disease (IBD)

\begin{tabular}{|c|c|c|c|c|c|}
\hline $\begin{array}{l}\text { Aim of } \\
\text { endoscopy }\end{array}$ & Type of IBD & $\begin{array}{l}\text { Target patient } \\
\text { group }\end{array}$ & Time point & Endpoint & Comment \\
\hline $\begin{array}{l}\text { Assessment of } \\
\text { mucosal } \\
\text { healing }\end{array}$ & $\mathrm{CD}$ and $\mathrm{UC}$ & $\begin{array}{l}\cdot \text { monitoring of } \\
\text { intensive therapy } \\
\text { - discrepancy between } \\
\text { symptoms and } \\
\text { laboratory results } \\
\text { - prior to cessation } \\
\text { of intensive therapy } \\
\text { - under neoplasia } \\
\text { surveillance }\end{array}$ & $\begin{array}{l}\text { by clinical } \\
\text { decision }\end{array}$ & $\begin{array}{l}\text { mucosal healing: } \\
\text { - UC: Mayo score } \\
0(-1) \\
\text { - CD: not clearly } \\
\text { defined, at least } \\
\text { absence of deep } \\
\text { ulcers }\end{array}$ & $\begin{array}{l}\text { - irritable bowel } \\
\text { syndrome may } \\
\text { persist despite } \\
\text { mucosal healing } \\
\text { - UC: sigmoidoscopy } \\
\text { often sufficient } \\
\text { - inflammation- } \\
\text { neoplasia sequence }\end{array}$ \\
\hline $\begin{array}{l}\text { Short-term } \\
\text { control of } \\
\text { response to } \\
\text { intensified } \\
\text { therapy }\end{array}$ & $\mathrm{UC}$ and $\mathrm{CD}$ & $\begin{array}{l}\text { - severe disease under } \\
\text { intensive therapy } \\
\text { - urgent surgery } \\
\text { contemplated }\end{array}$ & $\begin{array}{l}\text { short-term } \\
\text { control after } \\
\text { initiation of new } \\
\text { therapy } \\
\text { (e.g. 3-7 days) }\end{array}$ & response & $\begin{array}{l}\text { UC: sigmoidoscopy } \\
\text { often sufficient }\end{array}$ \\
\hline $\begin{array}{l}\text { Postoperative } \\
\text { surveillance }\end{array}$ & $\mathrm{CD}$ & $\begin{array}{l}\text { - after resection } \\
\text { in } \mathrm{CD}\end{array}$ & $\begin{array}{l}(3-) 6(-12) \text { months } \\
\text { postoperatively }\end{array}$ & Rutgeerts score & $\begin{array}{l}\text { endoscopic } \\
\text { recurrence precedes } \\
\text { and predicts clinical } \\
\text { recurrence }\end{array}$ \\
\hline $\begin{array}{l}\text { Neoplasia } \\
\quad \text { surveillance }\end{array}$ & $\begin{array}{l}\text { UC and } \\
\text { Crohn's } \\
\text { colitis }\end{array}$ & $\begin{array}{l}\text { - long-standing UC } \\
\text { or Crohn's colitis } \\
\text { - risk factors (PSC, } \\
\text { family history, ...) }\end{array}$ & $\begin{array}{l}-8 \text { years after first } \\
\text { manifestation } \\
\text { yearly to biennial } \\
\text { - PSC: after } \\
\text { diagnosis of PSC, } \\
\text { yearly }\end{array}$ & $\begin{array}{l}\text { - assessment of } \\
\text { mucosal healing } \\
\text { - detection and } \\
\text { endoscopic } \\
\text { resection of } \\
\text { precursor lesions } \\
\text { - early detection of } \\
\text { colitis-associated } \\
\text { cancer }\end{array}$ & $\begin{array}{l}\text { - chromoendoscopy } \\
\text { is superior to white } \\
\text { light endoscopy } \\
\text { - intensified } \\
\text { surveillance after } \\
\text { liver transplantation } \\
\text { for PSC }\end{array}$ \\
\hline
\end{tabular}

$\mathrm{CD}=$ Crohn's disease; $\mathrm{UC}=$ ulcerative colitis; $\mathrm{PSC}$ = primary sclerosing cholangitis. in UC patients in clinical remission [3]. Recently, a novel score for the description of subtle alterations in UC (PICaSSO) [4] has aimed to refine the endoscopic definition of mucosal healing, and further validation is ongoing at present.

In $\mathrm{CD}$, there is no validated definition of mucosal healing [5]. The simple endoscopic score (SES-CD) and the CD endoscopic index of severity (CDEIS) are well established in a trial setting; however, they require post-procedure time in routine practice and are limited to the evaluation of luminal CD manifestation. Thus, on the one hand, the multitude of different endoscopic scores implies that there may not be a 'one-size-fits-all' evaluation system, and on the other hand, this stresses the need for exact description and evaluation of lesions during surveillance endoscopy for mucosal healing. This is especially important since mucosal healing has become an important goal to achieve not only in clinical trials but also in daily practice $[5,6]$. With confocal endomicroscopy, even the barrier function can be visualized. Translational trials have linked the visualization of an impaired barrier function to the risk of recurrence even in macroscopically and histologically normal mucosa [7]. In clinical routine, faecal levels of leukocyte markers (calprotectin, lactoferrin) may reduce the need for or frequency of endoscopy for reassessing response at the mucosal level.

It seems biologically plausible that mucosal healing beneficially impacts on long-term prognosis in CD and UC. Still, the implica-
Fig. 1. a In a patient with a severe Crohn's disease flare, anti-TNF therapy was initiated after visualization of mucosal inflammation b 1 month later, the patient showed significantly less inflammation under continued therapy, and the ulcers have healed. c 7 months after initiation of therapy, stigmata of active inflammation were no longer seen. This is generally considered mucosal healing although signs of past inflammation (pseudopolyps) are still present.

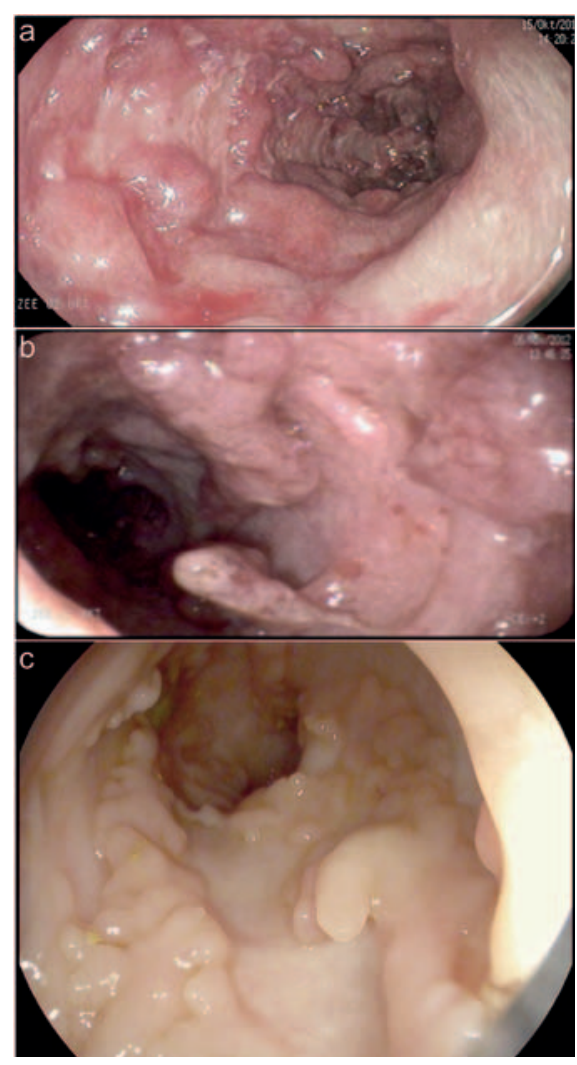


Fig. 2. a In steroid-refractory UC, b no endoscopic response was seen after 5 days of intravenous ciclosporin therapy. This patient eventually underwent proctocolectomy. c In a patient with UC refractory to anti-TNF/azathioprine combination therapy, $\mathbf{d}$ endoscopic response was seen 7 days after initiation of intravenous tacrolimus: healing ulcerations were paralleled by clinical response.

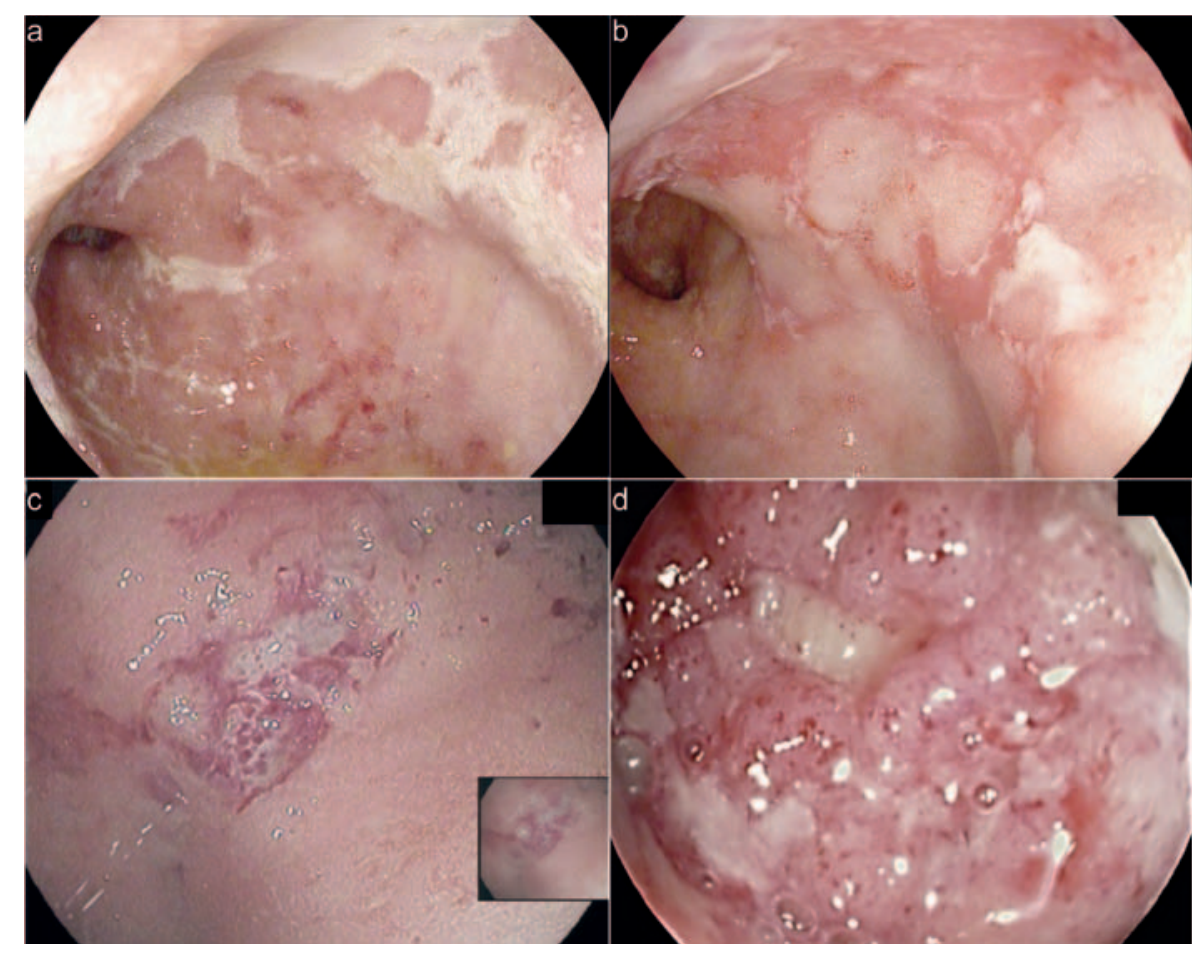

tions of intensified therapy of endoscopic inflammation in clinically quiescent disease ('treat to target' beyond clinical remission) have yet to be determined and formally investigated. In clinical routine, we are usually more inclined towards endoscopic surveillance of mucosal healing in more severe disease and more intensive therapy (fig. 1), and combine endoscopic re-evaluation with histologic assessment.

Another frequent clinical indication to re-evaluate mucosal healing may be when termination of immunosuppression is considered. Among other variables, endoscopically quiescent disease has been associated with a lower relapse rate after infliximab had been stopped [8]. We are more liberal towards terminating immunosuppression when the mucosal lesions are completely healed (fig. 1).

\section{Short-Term Control of Response to Intensified Therapy}

We follow a similar approach in patients with severe disease under intensified therapy. In severe steroid-refractory colitis, patients are usually put on infliximab or ciclosporin or tacrolimus therapy. Our interdisciplinary approach recommends short-term control of response to therapy in order to further stratify patients towards ongoing medical therapy or colectomy/resection. Figure 2 shows 2 patients undergoing tacrolimus and ciclosporin therapy in steroid-refractory severe UC. Usually at day 5-7 we complement repeat close clinical assessment with endoscopic re-evaluation. Not surprisingly, studies were able to correlate the endoscopic severity of disease with mid-term outcome $[9,10]$, although most trials have used a later time point (e.g. 12 weeks) for reassessment.
Fluorescent labelling of adalimumab has allowed endomicroscopic visualization of binding of the agent to its cellular target. This was predictive of response to therapy in a pilot trial [11].

\section{Postoperative Surveillance}

Ileocolonoscopy is the gold standard for the detection of postoperative recurrence in CD. After ileocaecal resection, endoscopic signs of recurrence can be frequently found at or proximal to the anastomosis in up to two-thirds of patients. Endoscopic recurrence usually precedes clinical recurrence; however, not every patient with inflammation at the anastomosis will develop a clinical flare. Therefore, we perform ileocolonoscopy 6-12 months after resection if findings translate into a change in clinical management or if pre-emptive treatment of recurrence has to be considered. The Rutgeerts score helps to predict recurrence [12]: A lower risk of recurrence has been found in patients without (i0) or only minimal (i1, less than 5 aphthous lesions) mucosal inflammation, or lesions confined to the anastomosis (i2a, even if more than 5 lesions or stenosis). A higher risk of recurrence that usually mandates therapy is found in patients with more than 5 aphthous lesions (i2b) and/or lesions in diffuse inflammation (i3) or ulcerations or inflammatory strictures (i4).

Surveillance after ileal pouch-anal anastomosis (IPAA) in UC patients is a matter of ongoing debate. Some authors recommend surveillance for pouchitis and for screening for neoplasia on a yearly basis (see below). We stratify patients according to indications for IPAA (refractory inflammation vs. neoplasia, the latter requiring more intensive surveillance), remnant rectal mucosa and/or performance of intraoperative mucosectomy, and symp- 


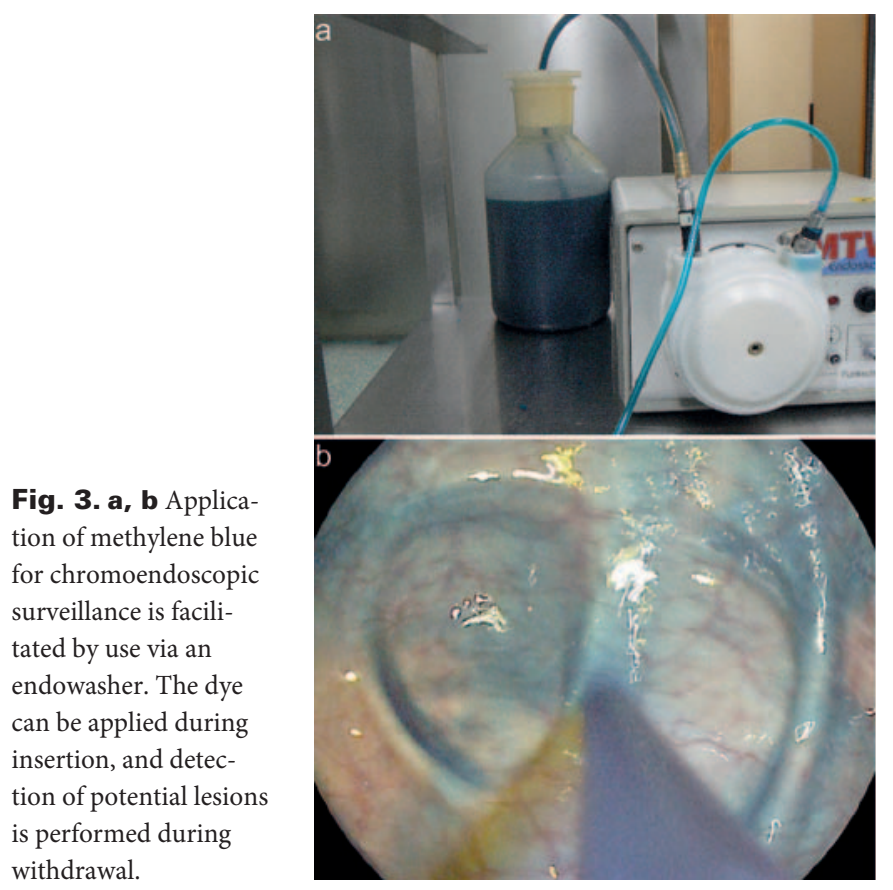

toms of the patient (e.g. chronic pouchitis). The same applies to patients with ileorectal anastomoses or Hartmann situation.

\section{Endoscopy for Neoplasia Surveillance}

Long-standing UC confers a higher risk to develop colitis-associated colorectal cancer (CRC) in comparison to the non-IBD population. The risk in Crohn's colitis is similar if adjusted for the affected mucosal surface. This means that Crohn's colitis mandates similar endoscopic surveillance if more than one colonic segment is affected [5]. The CRC risk correlates with the extent and activity of the inflammation as well as with the presence of primary sclerosing cholangitis (PSC) and family history of CRC.

Patients with colitis are included in an endoscopic surveillance programme starting from 8 years after initial manifestation (not: after initial diagnosis!). Patients with PSC should undergo yearly colonoscopy starting from the diagnosis of PSC. Patients with isolated ulcerative proctitis and $\mathrm{CD}$ patients with only one affected colonic segment (e.g. caecum) are at no increased risk to develop CRC. Pseudopolyps are no longer considered a specific risk factor by themselves but rather indicate long-standing inflammation and may interfere with thorough inspection during endoscopy. The colonoscopy frequency has not been formally evaluated. According to the risk factors (see above), the German guidelines [6] favour yearly to biennial intervals. The European Crohn's and Colitis Organisation (ECCO) guideline [5] further stratifies high-risk from intermediate-risk and low-risk groups with respective surveillance intervals (annual surveillance vs. surveillance every 2-3 years vs. surveillance every 5 years).

Surveillance colonoscopy is best performed in a non-inflamed colon after meticulous bowel preparation. Both endoscopically and histologically, differentiation of inflammatory reactive changes from early neoplasia can be challenging. However, surveillance should not be postponed indefinitely if mucosal healing cannot be achieved. Time requirements for withdrawal are higher than for normal screening colonoscopy, and 6 min will certainly not suffice, given the often subtle and flat nature of colitis-associated dysplasia.

Former and some current guidelines recommend surveillance with extensive random biopsies from all colonic segments. This recommendation is mainly based on older evidence from pre-high definition endoscopy studies. Newer guidelines (ECCO 2013 [5]; German Society for Gastroenterology, Digestive and Metabolic Diseases (DGVS), in press) have paid tribute to the intensive discussion on time requirements and low diagnostic yield of random biopsies [13], and the fact that most even flat dysplasia is visible during thorough examination $[14,15]$. Use of high-definition endoscopes is indispensable. For high-definition endoscopy, retrospective trials suggest higher prevalence ratios of 2.21 (confidence interval (CI) 1.09-4.45) for detecting any dysplastic lesion and of 2.99 (CI 1.16-7.79) for detecting dysplastic lesions on targeted biopsy [16]. Larger prospective trials have been suggested; however, high definition is the standard in most endoscopy suites, so formal confirmation - although scientifically mandated - will no longer be necessary to change clinical practice accordingly. Currently, there is no consensus as to whether obtaining random biopsies from normal mucosa in high-definition endoscopy should be preferred to targeted biopsies only [17]. A recent prospective, randomized trial on 246 patients with long-standing UC in 52 Japanese centres suggested that high-definition white light endoscopy (WLE) alone with targeted biopsies only shows a trend toward superior detection of dysplasia when compared to WLE with random biopsies [18]. The fact that detection is better if no random biopsies are obtained is surprising at first sight, since in both study arms high-definition white light endoscopes have been used. The authors attribute this to the longer time spent on scrutinizing the mucosa (instead of biopsy handling) and a better view not obscured by post-biopsy bleeding. This also suggests that most neoplasia might be visible on thorough examination despite its flat appearance, which has been one of the reasons to promote random biopsies in the pre-high definition era $[14,15]$.

Detection rates can be further optimized by pan-chromoendoscopy, i.e. the application of intravital stains onto the mucosa in order to enhance the contrast between normal and pathologic mucosa. Both methylene blue (0.1\%) and indigo carmine (0.1-0.5\%) have been studied (see review in [17]). The original use of chromoendoscopy surveillance in UC was carried out via a spraying catheter [19]. For ease of use, we mostly apply methylene blue dilution via the endowasher (fig. 3) so that during insertion, cleaning and staining of the mucosa are performed simultaneously. What is more, chromoendoscopy does not require additional time but actually allocates time to thorough inspection instead of random biopsies, which can be omitted if chromoendoscopy is used (see below). The dilution has to be strong enough to stain the mucosa appropriately; however, visualization of vessels should not be obscured by too intensive staining. For focal staining and exact clas- 


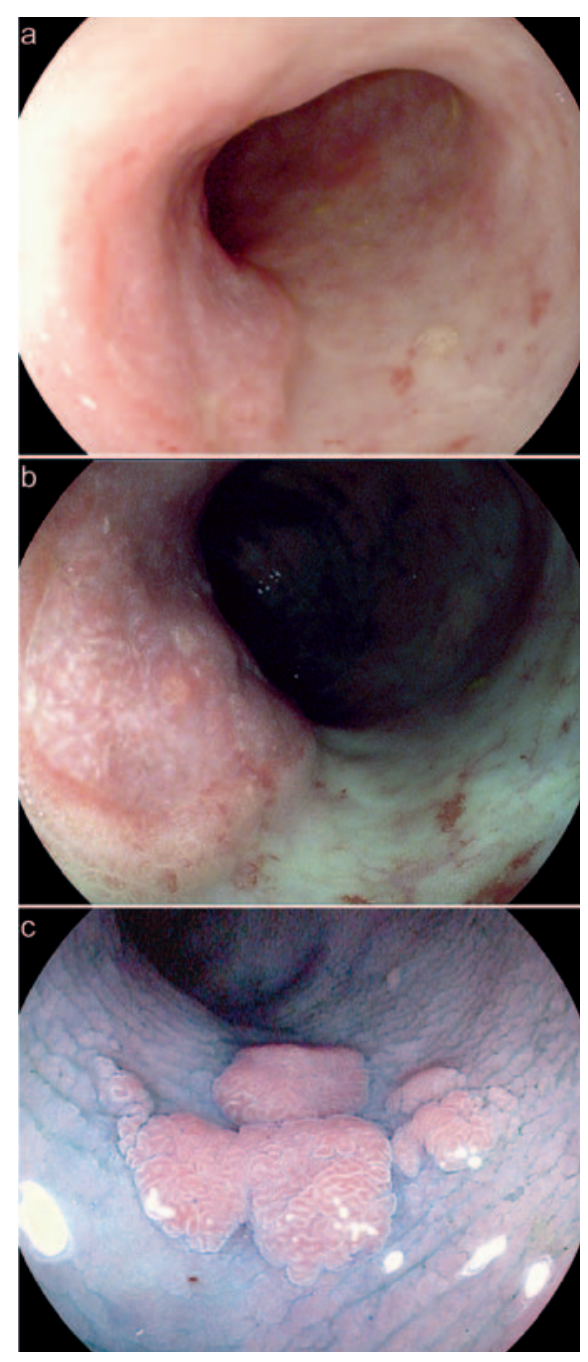

Fig. 4. a High-definition white light endoscopy shows a suspicious ulcerous lesion in a severely atrophic colon in long-standing ulcerative colitis. b Virtual chromoendoscopy with i-scan clearly identifies the margins and pathologic vascular structure of this dysplastic lesion. Targeted biopsies confirmed the suspected colitis-associated cancer. c Chromoendoscopy with methylene blue highlights the borders of a confirmed low-grade intraepithelial neoplasia. The lesion was completely resected endoscopically.

sification of suspicious lesions, higher concentrations can be used if necessary.

A recent meta-analysis confirmed the superior diagnostic yield of chromoendoscopy for neoplasia when compared to WLE with quadrant biopsies [20]. The 'number-needed-to-stain' was 14 patients to detect 1 additional patient with neoplasia. In a meta-analysis of six randomized studies, sensitivity and specificity for chromoendoscopy for intraepithelial neoplasia were 83.3 and $91.3 \%$, respectively [21]. Chromoendoscopy required additional examination time and was operator-dependent. Still, chromoendoscopy with targeted biopsies only is cost-effective [22]. It is not necessary to obtain random biopsy specimens from chromoendoscopically normal-appearing mucosa according to European [5] or German surveillance recommendations.

Virtual chromoendoscopy and optical or post-image acquisition filtering (narrow band imaging (NBI), i-scan, FICE, SPICE) seem to be attractive alternatives. They provide optical enhancement techniques by pressing a button on the handpiece of the endoscopes, come along without time requirements, and are fully reversible. However, they have not (yet) been able to replicate the good results of chromoendoscopy comprehensively. Whether this
Fig. 5. a In a patient with Crohn's disease, a suspicious area was biopsied at the ileo-ascendostomy. Although low-grade neoplasia was histologically confirmed, resection was not performed for different reasons. b 6 months later, the lesion had increased in size. Surgical resection yielded submucosal adenocarcinoma with intact, non-neoplastic mucosal cover.

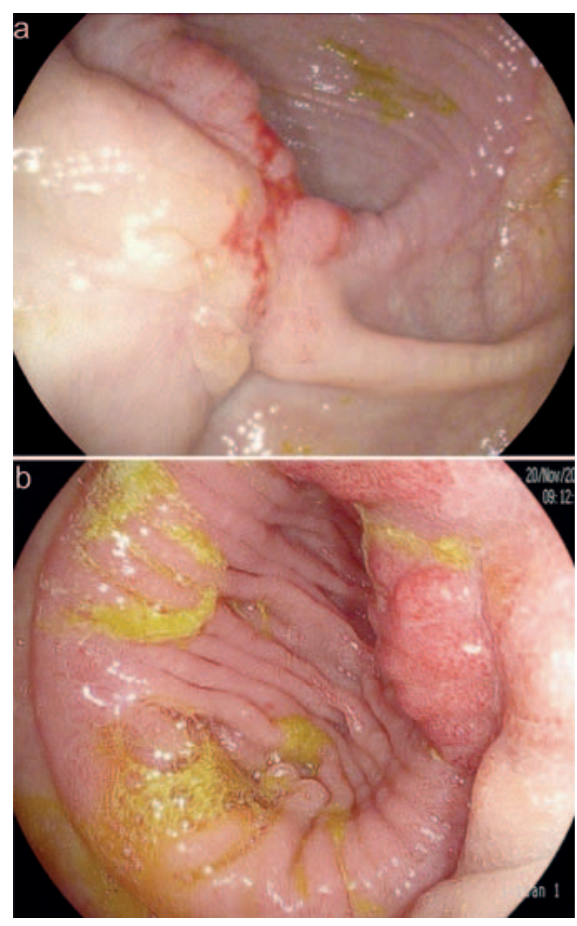

is due to the lower light intensity of the 'earlier' generation virtual techniques or due to the fact that chromoendoscopy preferentially enhances crypt patterns, whereas virtual techniques enhance vessels, is not entirely clear. In a parallel group randomization design, no difference in dysplasia detection was noted [23]. A trend toward more suspicious lesions detected by NBI was accompanied by a higher false-positive rate. Similarly, in a randomized delayed crossover design, no difference in dysplasia detection was found [24]. When NBI was compared to chromoendoscopy in 60 patients in a prospective, randomized, crossover design, both techniques showed similar true positive rates for 13 intraepithelial lesions and a lower false-positive rate for NBI, but a trend toward a higher percentage of missed lesions with NBI [25]. As expected, the withdrawal time was lower with NBI (16 vs. 27 min with chromoendoscopy). In summary, optical enhancement techniques are not recommended for the replacement of chromoendoscopy $[5,17]$.

When lesions are detected, the main decision is whether the lesion is endoscopically resectable or not (fig. 4). The former differentiation of DALM (dysplasia-associated lesional mass) versus ALM (adenoma-like mass) (terms that are no longer used for decision making in the current guidelines $[5,17])$ and biopsy results of highgrade versus low-grade dysplasia do not interfere with the decision to resect, if technically feasible. An endoscopic resection is safe for circumscript lesions that are resected completely, irrespective of both low-grade or high-grade dysplasia and of the localisation relative to the inflammation (the latter may be subject to change in the course of disease and therapy) (fig. 4c) [26-30]. Importantly, these patients are at increased risk for simultaneous or metachronous intraepithelial neoplasia. Close follow-up surveillance with high-definition chromoendoscopy by an experienced IBD endoscopist is recommended as a first-line option for these patients $[5,17]$. Patients 
can be counselled about proctocolectomy if careful surveillance is no option. Resection and submission of a large piece of tissue for histopathologic evaluation is also highly relevant from a diagnostic point of view. Superficial biopsies may result in underestimating the grade of dysplasia and the depth of invasion. A significant percentage of colitis-associated neoplastic lesions shows a 'bottom-up' morphology with low-grade neoplasia in superficial biopsies and higher grades in deeper sections (fig. 5). Up to $11 \%$ of IBD-associated cancers have been attributed to these so-called intestinal low-grade tubulo-glandular adenocarcinomas [31]. These may not be detected on biopsies alone but usually require a larger specimen for diagnosis. mucosal healing and short-term control of response to intensified therapy. For postoperative surveillance in $\mathrm{CD}$, mucosal changes (as graded by the Rutgeerts Score) are predictive of clinical recurrence. For neoplasia surveillance, virtual chromoendoscopy has not (yet) replaced conventional chromoendoscopy. Surveillance can be optimized by the use of high-definition endoscopes as well as (pan-) chromoendoscopy and requires time to scrutinize the mucosa. This seems superior to spending time on taking random biopsies. Lesions amenable to endoscopic resection should be treated endoscopically.

\section{Disclosure Statement}

\section{Conclusion}

Endoscopy is a key technique for the surveillance of patients with IBD. It is the gold standard in the long-term assessment of
No conflict of interest.

\section{References}

1 Schroeder KW, Tremaine WJ, Ilstrup DM: Coated oral 5-aminosalicylic acid therapy for mildly to moderately active ulcerative colitis. A randomized study. N Engl J Med 1987;317:1625-1629.

2 Travis SP, Schnell D, Krzeski P, et al: Developing an instrument to assess the endoscopic severity of ulcerative colitis: the Ulcerative Colitis Endoscopic Index of Severity (UCEIS). Gut 2012;61:535-542.

3 Arai M, Naganuma M, Sugimoto S, et al: The Ulcerative Colitis Endoscopic Index of severity is useful to predict medium- to long-term prognosis in ulcerative colitis patients with clinical remission. J Crohns Colitis 2016;10:1303-1309.

4 Iacucci M, Daperno M, Lazarev M, et al: Development and reliability of the new endoscopic virtual chromoendoscopy score: the PICaSSO (Paddington International Virtual ChromoendoScopy ScOre) in ulcerative colitis. Gastrointest Endosc 2017;86:1118-1127.e5.

5 Annese V, Daperno M, Rutter MD, et al: European evidence based consensus for endoscopy in inflammatory bowel disease. J Crohns Colitis 2013;7:982-1018.

6 Dignass A, Preiss JC, Aust DE, et al: Updated German guideline on diagnosis and treatment of ulcerative colitis, 2011 (Article in German). Z Gastroenterol 2011;49: 1276-1341.

7 Kiesslich R, Duckworth CA, Moussata D, et al: Local barrier dysfunction identified by confocal laser endomicroscopy predicts relapse in inflammatory bowel disease. Gut 2012;61:1146-1153.

8 Louis E, Mary JY, Vernier-Massouille G, et al: Maintenance of remission among patients with Crohn's disease on antimetabolite therapy after infliximab therapy is stopped. Gastroenterology 2012;142:63-70.e5; quiz e31.

9 Miyoshi J, Matsuoka K, Inoue N, et al: Mucosal healing with oral tacrolimus is associated with favorable medium- and long-term prognosis in steroid-refractory/ dependent ulcerative colitis patients. J Crohns Colitis 2013;7:e609-614.

10 Ikeya $\mathrm{K}$, Hanai $\mathrm{H}$, Sugimoto $\mathrm{K}$, et al: The Ulcerative Colitis Endoscopic Index of Severity more accurately reflects clinical outcomes and long-term prognosis than the Mayo endoscopic score. J Crohns Colitis 2016;10:286-295.
11 Atreya R, Neumann H, Neufert C, et al: In vivo imag ing using fluorescent antibodies to tumor necrosis fac tor predicts therapeutic response in Crohn's disease. Nat Med 2014;20:313-318.

12 Rutgeerts P, Geboes K, Vantrappen G, et al: Predictability of the postoperative course of Crohn's disease. Gastroenterology 1990;99:956-963.

13 van den Broek FJ, Stokkers PC, Reitsma JB, et al: Random biopsies taken during colonoscopic surveillance of patients with longstanding ulcerative colitis: low yield and absence of clinical consequences. Am J Gastroenterol 2014;109:715-722.

14 Rutter MD, Saunders BP, Wilkinson KH, et al: Most dysplasia in ulcerative colitis is visible at colonoscopy. Gastrointest Endosc 2004;60:334-339.

15 Rubin DT, Rothe JA, Hetzel JT, et al: Are dysplasia and colorectal cancer endoscopically visible in patients with ulcerative colitis? Gastrointest Endosc 2007;65: 998-1004.

16 Subramanian V, Ramappa V, Telakis E, et al: Comparison of high definition with standard white light endoscopy for detection of dysplastic lesions during surveillance colonoscopy in patients with colonic inflammatory bowel disease. Inflamm Bowel Dis 2013;19:350355.

17 Laine L, Kaltenbach T, Barkun A, et al: SCENIC international consensus statement on surveillance and management of dysplasia in inflammatory bowel disease. Gastrointest Endosc 2015;81:489-501.e26.

18 Watanabe T, Ajioka Y, Mitsuyama K, et al: Comparison of targeted vs random biopsies for surveillance of ulcerative colitis-associated colorectal cancer. Gastroenterology 2016;151:1122-1130.

19 Kiesslich R, Fritsch J, Holtmann M, et al: Methylene blue-aided chromoendoscopy for the detection of intraepithelial neoplasia and colon cancer in ulcerative colitis. Gastroenterology 2003;124:880-888.

20 Subramanian V, Mannath J, Ragunath K, et al: Metaanalysis: the diagnostic yield of chromoendoscopy for detecting dysplasia in patients with colonic inflammatory bowel disease. Aliment Pharmacol Ther 2011;33: 304-312.
21 Wu L, Li P, Wu J, et al: The diagnostic accuracy of chromoendoscopy for dysplasia in ulcerative colitis: meta-analysis of six randomized controlled trials. Colorectal Dis 2012;14:416-420.

22 Konijeti GG, Shrime MG, Ananthakrishnan AN, et al: Cost-effectiveness analysis of chromoendoscopy for colorectal cancer surveillance in patients with ulcerative colitis. Gastrointest Endosc 2014;79:455-465.

23 Ignjatovic A, East JE, Subramanian V, et al: Narrow band imaging for detection of dysplasia in colitis: a randomized controlled trial. Am J Gastroenterol 2012; 107:885-890.

24 van den Broek FJ, Fockens P, van Eeden S, et al: Narrow-band imaging versus high-definition endoscopy for the diagnosis of neoplasia in ulcerative colitis. Endoscopy 2011;43:108-115.

25 Pellise M, Lopez-Ceron M, Rodriguez de Miguel C, et al: Narrow-band imaging as an alternative to chromoendoscopy for the detection of dysplasia in longstanding inflammatory bowel disease: a prospective, randomized, crossover study. Gastrointest Endosc 2011;74:840-848.

26 Engelsgjerd M, Farraye FA, Odze RD: Polypectomy may be adequate treatment for adenoma-like dysplastic lesions in chronic ulcerative colitis. Gastroenterology 1999;117:1288-1294; discussion 1488-1491.

27 Rubin PH, Friedman S, Harpaz N, et al: Colonoscopic polypectomy in chronic colitis: conservative management after endoscopic resection of dysplastic polyps. Gastroenterology 1999;117:1295-1300.

28 Odze RD, Farraye FA, Hecht JL, et al: Long-term follow-up after polypectomy treatment for adenoma-like dysplastic lesions in ulcerative colitis. Clin Gastroenterol Hepatol 2004;2:534-541.

29 Vieth M, Behrens H, Stolte M: Sporadic adenoma in ulcerative colitis: endoscopic resection is an adequate treatment. Gut 2006;55:1151-1155.

30 Blonski W, Kundu R, Furth EF, et al: High-grade dysplastic adenoma-like mass lesions are not an indication for colectomy in patients with ulcerative colitis. Scand J Gastroenterol 2008;43:817-820.

31 Levi GS, Harpaz N: Intestinal low-grade tubuloglandular adenocarcinoma in inflammatory bowel disease. Am J Surg Pathol 2006;30:1022-1029. 